\title{
Research on Improved IPSO-LSSVM Method and Its Application
}

\author{
Peng-fei LIU ${ }^{1}$, Qun-tai SHEN ${ }^{1}$ and Jun ZHII,* \\ ${ }^{1}$ School of Information Science and Engineering, Central South University, China \\ ${ }^{2}$ Beijing institute of remote sensing information, China \\ ${ }^{*}$ Corresponding author
}

Keywords: Particle Swarm Optimization, Support Vector Machine, Cost Prediction

\begin{abstract}
It adopts support vector machine which is applicable for small sample prediction and constructs the prediction model. It is based on analyzing characteristics of particle swarm optimization and support vector machine. The improved IPSO-LSSVM prediction model shall be used to predict the development cost of military excavator. The prediction result indicates that compared with traditional SVM algorithm and BP algorithm, the prediction model has a better small sample adaptability, a faster training velocity and a higher prediction accuracy and it is more applicable to predict the development cost of engineering equipment.
\end{abstract}

\section{Introduction}

The engineering equipment has a long preparation time, a difficult technology, and many uncertain factors. Many costs in the developing process are hard to get and related costs and data can be obtained after costs take place. A few effective training samples become a key problem in affecting the cost prediction.

To solve above problems effectively, systematic and scientific analytical method needs to be adopted and the development cost of engineering equipment shall be predicted in accordance with historical data which is of typical significance. Because it is hard to collect abundant effective sample data and factors which affect the development cost of equipment are nonlinear, so it has limitations to predict the development cost of equipment using traditional parametric method [1]. Support vector machine has a strong ability to deal with nonlinear problems and it is applicable to small sample data. Therefore, the development cost of engineering equipment shall be predicted by using improved support vector machine.

\section{Prediction Model Based on Improved IPSO-LSSVM}

\section{Adaptive Inertia Factor Strategy}

It can be seen that the initial stage of iteration determines global searching ability of algorithm, the final stage determines local searching ability [2], if search time of both ends can be lengthened, it is of great benefit to the improvement of algorithm performance, and the weight of the following can be adopted to self-adopt update method:

$$
\begin{aligned}
& w=w_{\min }+\left(w_{\max }-w_{\min }\right) \times \exp \left(-20 \times\left(t / t_{\max }\right)^{6}\right) \\
& w_{\max }, w_{\min } \text { takes } 0.9 \text { and } 0.1 \text { separately. }
\end{aligned}
$$

This method makes inertia weight keep a larger value for a long time at the beginning of iteration, it keeps a smaller value for a long time at the end of iteration, thus the global search time in early 
iterations and the local search time in late iterations will be added, the intensity of global search in early iterations and the intensity of local search in late iterations will be strengthened, keeping global search ability and local search ability in good balance.

\section{Mutation Strategy With No Evolution Generation}

(1)Calculate fitness rate: fitness rate refers to fitness rate of historically optimal location of particle $p_{g}$ on this time iteration and $M$ times iterations before this time (M is 1):

$$
\text { FSlope }=\frac{f\left(p_{g}(t)\right)-f\left(p_{g}(t-M)\right)}{f\left(p_{g}(t)\right)}
$$

(2)Statistics of no evolutionary times: When the evolution begins, no evolutionary times stop time shall be 0 , fitness change threshold Slope value (take 10e-10), no evolutionary threshold value of particles Max step and mutation probability $p_{m}$ (take 0.4).In the process of iteration, no evolutionary times of particles shall be decided by fitness rate, as shown below:

$$
\left\{\begin{array}{lr}
\text { FSlope }<\text { Slopevalue } & \text { Stoptime }=\text { stoptime }+1 \\
\text { FSlope }>\text { Slopevalue } & \text { Stoptime }=0
\end{array}\right.
$$

If no evolutionary times have exceeded set no evolutionary threshold value, the algorithm may be in a stage of stagnation, the population shall implement mutation operation according to mutation probability:

$$
p_{g i}(t)=p_{g i}(t)+0.5 \times \operatorname{rand}() \times p_{g i}(t)
$$

The improvement make IPSO avoid phenomenon that it falls into local minimum and the training stops when training, when the training stagnates, algorithm makes the population particle jump out of local minimum point by mutation operation and keeps drawing close to global optimum point.

\section{LSSVM Deformation Strategy}

According to the method of transforming standard SVM into duality problem [3], LSSVM primal problem can be transformed into duality problem by derivation:

$$
\begin{aligned}
& \frac{\partial L}{\partial \omega}=0 \rightarrow \omega=\sum_{i=1}^{n} \alpha_{i} \phi\left(x_{i}\right) \\
& \frac{\partial L}{\partial b}=0 \rightarrow \sum_{i=1}^{n} \alpha_{i}=0 \\
& \frac{\partial L}{\partial \xi_{i}}=0 \rightarrow \alpha_{i}=\gamma \xi_{i}, \gamma=2 C \\
& \frac{\partial L}{\partial \alpha_{i}}=0 \rightarrow \omega^{T} \phi\left(x_{i}\right)+b+\xi_{i}+\varepsilon-y_{i}=0
\end{aligned}
$$

After eliminating $\omega$ and $\xi$ : 
$\left\{\begin{array}{c}\frac{\partial L}{\partial b}=0 \rightarrow \sum_{i=1}^{n} \alpha_{i}=0 \\ \frac{\partial L}{\partial \alpha}=0 \rightarrow \sum_{i=1}^{n} \alpha_{i} K\left(x_{i}, x_{j}\right)+b+\varepsilon+\frac{\alpha_{i}}{\gamma}-y_{i}=0\end{array}\right.$

Transforming into matrix form:

$$
\left[\begin{array}{cccc}
0 & 1 & \cdots & 1 \\
1 & K\left(x_{1}, x_{1}\right)+2 / c & \cdots & K\left(x_{1}, x_{n}\right) \\
\vdots & \vdots & \cdots & \vdots \\
1 & K\left(x_{n}, x_{1}\right) & \cdots & K\left(x_{n}, x_{n}\right)+2 / c
\end{array}\right] \bullet\left[\begin{array}{c}
b+\varepsilon \\
\alpha_{1} \\
\vdots \\
\alpha_{n}
\end{array}\right]=\left[\begin{array}{c}
0 \\
y_{1} \\
\vdots \\
y_{n}
\end{array}\right]
$$

Decision function is:

$$
f(x)=\sum_{i=1}^{n} \alpha_{i} K\left(x_{i}, x\right)+\bar{b}
$$

Because LSSVM is solving linear equation groups, $\bar{b}$ in decision function can be acquired by equation [4]. Deformed prediction model is simpler in form, more simplified in calculation and less calculation.

\section{Prediction Analysis of Engineering Equipment's Development Cost}

It uses the improved IPSO-LSSVM prediction model to predict the military excavator's development cost; check the usability of prediction model in predicting engineering equipment's development cost. This method also applies to other general engineering equipment.

\section{Pretreatment of Sample Data}

When predicting engineering equipment's development cost, cost is dependent variable and characteristic parameter which affects cost is independent variable. On the one hand choosing characteristic shall not miss important affecting factor and choose the characteristic parameter which has higher correlation to development cost; on the other hand, the principle of parameter minimalists needs to be followed, the expressed characteristic parameter shall be reduced. Therefore, characteristic attribute of engineering equipment shall be deducted using correlation method before prediction, keep characteristic parameter which has larger relation to cost and independent of each other as driving factor of development cost.

\section{Forecasting Process and Accuracy Check of Model}

The training sample data after normalization in above tables shall be input into prediction model of BP neural network, traditional SVM prediction model, the improved IPSO-LSSVM prediction model for training. Among them, BP prediction model adopts BP prediction model with momentum factor and the chosen model's hidden node numbers are 18, training target is 0.00001 , traditional SVM prediction model's parameter $\mathrm{C}=300$ [5], nuclear parameter is 5, nuclear parameters of SVM prediction model and improved IPSO-LSSVM prediction model all adopt RBF nuclear parameter, such as equation(12).

$$
K\left(x_{i}, x_{j}\right)=\exp \left(-\frac{\left|x_{i}-x_{j}\right|^{2}}{2 \sigma^{2}}\right)
$$




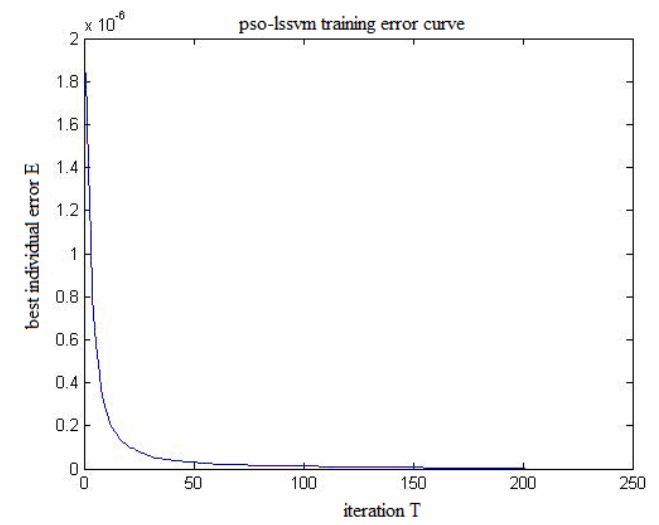

Fig 1 Error curve of using improved IPSO to train the LSSVM

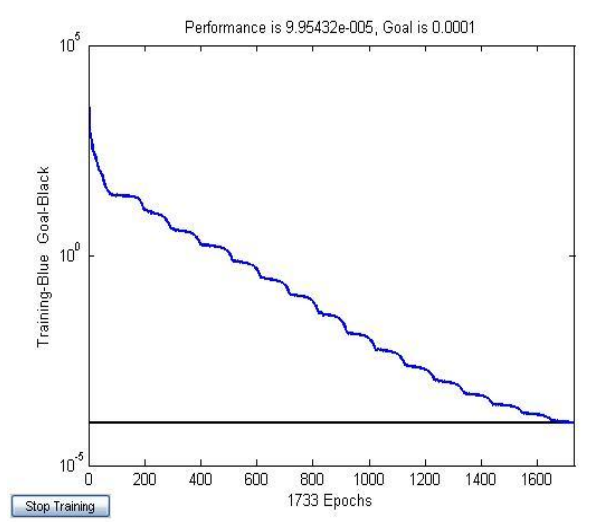

Fig 2 Error curve of BP network

Above figures are training error curve figures of IPSO-LSSVM prediction model and BP prediction model, it is known from figures: training error of improved IPSO-LSSVM prediction model declines rapidly and it tends to stop after 200 generation training; but BP prediction model does not meet the training requirement after 1733 generation training, the training efficiency of improved IPSO-LSSCM prediction model is obviously higher than BP prediction model.

After the training, three prediction methods will respectively get prediction models applicable to sample data. The prediction sample shall be input into prediction models for forecasting. Because initial parameter has a great influence on BP prediction model, to lower the randomness of prediction, ten times prediction shall be continuously implemented on BP network to calculate the average value, average percentage error of prediction results shall be calculated, prediction results and error comparison are shown in Table 1:

Table 1 Correlation of three prediction models' prediction results

\begin{tabular}{ccccc}
\hline $\begin{array}{c}\text { prediction } \\
\text { sample }\end{array}$ & $\begin{array}{c}\text { true } \\
\text { value }\end{array}$ & $\begin{array}{c}\text { BP prediction } \\
\text { model }\end{array}$ & $\begin{array}{c}\text { SVM prediction } \\
\text { model }\end{array}$ & $\begin{array}{c}\text { Improved IPSO-LSSVM prediction } \\
\text { model }\end{array}$ \\
\hline 1 & 53.3 & 55.2302 & 54.5029 & 52.7725 \\
2 & 88.3 & 89.4308 & 87.8888 & 88.1401 \\
3 & 94.5 & 98.5698 & 97.8723 & 95.9778 \\
\hline
\end{tabular}

Table 2 Prediction error contrast of three prediction models

\begin{tabular}{cccc}
\hline prediction sample & BP prediction model & SVM prediction model & $\begin{array}{c}\text { improved IPSO-LSSVM } \\
\text { prediction model }\end{array}$ \\
\hline 1 & $3.62 \%$ & $2.27 \%$ & $0.98 \%$ \\
2 & $1.28 \%$ & $0.47 \%$ & $0.18 \%$ \\
3 & $4.31 \%$ & $3.57 \%$ & $1.56 \%$ \\
\hline MPAPE & $3.07 \%$ & $2.1 \%$ & $0.91 \%$ \\
\hline
\end{tabular}

\section{Conclusions}

Improved IPSO-LSSVM prediction model has used optimized model parameter and nuclear parameter, prediction accuracy of improved IPSO-LSSVM prediction model is much better than un-optimized SVM prediction model and BP prediction model. Improved IPSO-LSSVM prediction model and SVM prediction model depend on their excellent generalization performance, when there are a few training samples, prediction accuracy is much higher than BP prediction model. This shows that improved IPSO-LSSVM prediction model are better than traditional SVM and BP 
prediction model in both training efficiency and prediction accuracy, and it is more applicable to predicting engineering equipment's development cost with fewer data.

\section{References}

[1]Zeng-ming Guo. Equipment Economics. Beijing: Statistics Press of China, 2006.

[2]Xiao-dong Duan. Particle Swarm Optimization and Its Applications. Shenyang: Liaoning University Press, 2007.

[3] Bassel Soudan, Mohamed Saad. An evolutionary dynamic population size PSO implementation. Proceeding of Information and Communication Technologies: From Theory to Application, 2008.

[4] Zhang Y N, Teng H F. Detecting particle swarm optimization. Concurrency and Computation-Practice and Experience, 2009.

[5]Nai-yang Deng. New Method in the Data Mining-Support Vector Machine. Beijing: Science Press, 2004. 